\title{
Sarcoidosis-Lymphoma Syndrome: A Spectrum of One Disease
}

\author{
Khalifa E. Sharquie*, Raafa K. Al-Hayani, Waqas S. Abdulwahhab, \\ Abd-Allah S. Mohammed
}

The Scientific Council of Dermatology and Venereology-Iraqi and Arab Board for Medical Specializations, Department of Dermatology and Venereology, Baghdad Teaching Hospital, College of Medicine, University of Baghdad,

Baghdad, Iraq

Email: ksharquie@ymail.com, raafahayani@yahoo.com

Received 7 July 2015; accepted 28 August 2015; published 31 August 2015

Copyright (C) 2015 by authors and Scientific Research Publishing Inc.

This work is licensed under the Creative Commons Attribution International License (CC BY). http://creativecommons.org/licenses/by/4.0/

(c) (i) Open Access

\begin{abstract}
Lymphoma might occur in association with sarcoidosis or sarcoidosis might be combined with lymphoma, in so called ("sarcoidosis-lymphoma syndrome"). This syndrome is well reported in medical literature. The question, which one starts before is difficult to answer, as in some cases sarcoidosis starts first then is followed by lymphoma but in other cases during the course of lymphoma sarcoid reaction might be seen. In the present report, we describe a case of 60 years male patient that is presented with a rash with classical sarcoid pathology that overtime changes into typical lymphoma ended with death.
\end{abstract}

\section{Keywords}

Sarcoidosis, Lymphoma, Granuloma

\section{Introduction}

Sarcoidosis is well known as a multisystem disease of unknown etiology characterized by the presence of noncaseating granuloma in affected organs. The disease may affect any organ but most commonly involves the lungs, skin, and eyes. Clinically, patients may be completely asymptomatic or experience a progressive clinical course [1].

The etiology of sarcoidosis remains unknown and the disease remains a diagnosis of exclusion. The current understanding regarding the pathogenesis of the disease involves the exposure of a genetically susceptible individual to some environmental antigenic stimulus. The genetic predisposition may affect not only the occurrence

${ }^{*}$ Corresponding author.

How to cite this paper: Sharquie, K.E., Al-Hayani, R.K., Abdulwahhab, W.S. and Mohammed, A.-A.S. (2015) SarcoidosisLymphoma Syndrome: A Spectrum of One Disease. Journal of Cosmetics, Dermatological Sciences and Applications, 5, 181188. http://dx.doi.org/10.4236/jcdsa.2015.53022 
of disease but also the clinical expression and course. To date, the precise nature of the environmental stimulus remains unknown, although numerous leads and theories have been investigated and continue to be explored. Despite these efforts, the ability to identify a specific cause for this disease remains elusive [2].

Treatment is usually designed to help relieve the symptoms and thus does not directly alter the course of the disease [3]. This treatment usually consists of anti-inflammatory drugs like ibuprofen or aspirin [3]. In cases where the condition develops to the point that it has a progressive and/or life-threatening course, the treatment is most often steroid treatment with prednisolone [4]. Alternatively, drugs that are most commonly used to treat cancer and suppress the immune system, such as methotrexate, azathioprine and leflunomide, may be used [3] [4].

The disease can remit spontaneously or become chronic, with exacerbations and remissions. In some persons, it can progress to pulmonary fibrosis and death. About half of cases resolve without treatment or can be cured within 12 - 36 months, and most within five years. Some cases, however, may persist several decades [4]. Twothirds of people with the condition achieve a remission within 10 years of the diagnosis. Overall mortality ranges from $1 \%-6 \%$ [1]. When the heart is involved, the prognosis is generally less favorable, although, corticosteroids appear effective in improving AV conduction [5].

The association between lymphoma and sarcoidosis was first suggested by Bichel and Brincker in the 1960s [6]. Since then, additional descriptions of this possible connection have been published but without providing a better understanding of this relationship [1]. The sarcoidosis-lymphoma syndrome descried by Brincker [7] [8] was established on the basis of 2 different studies. In a Danish registry of patients with respiratory sarcoidosis, lymphoma occurred 11.5 times more frequently than expected in the general population [8].

Second, Brincker [7] reviewed 46 cases of lymphoma occurring in patients with sarcoidosis. He reported only 2 cases in which lymphoma preceded sarcoidosis with an additional 2 cases in a subsequent study [9]. Sarcoidosis patients are about $40 \%$ - 60\% more likely to develop malignancy, including solid tumors such as non-melanoma skin cancers (three-fold risk), renal cancer, and nonthyroid endocrine tumors [10].

Cases have been published in which sarcoidosis precedes lymphoma and vice-versa, albeit they can infrequently appear simultaneously. Sarcoidosis may precede the development of a lymphoma (the "sarcoidosislymphoma syndrome") by 18 months to 28 years [11]. All types of lymphoma may develop, and there is an increased incidence of thyroid cancer and leukaemia [12].

In the present case report, we are describing a patient that has first sarcoidosis and then changes into lymphoma. The aim of present report is to describe atypical spectrum of sarcoidosis-lymphoma state. The consent form taken from patient's family is about the publication of his condition.

\section{Case Report}

We present the case of a 60 years old male patient who was being referred to the dermatological department in Baghdad Teaching Hospital from Tikrit Teaching Hospital suspecting Kaposi sarcoma and complaining of recurrent, multiple, mildly itchy skin lesions all over the body mainly on the extremities for 3 years duration. No other systemic complaints were mentioned. Physical examination revealed multiple, diffuse, well-defined brown nodules and plaques distributed all over the body excluding the face, palms, soles, scalp, and genitalia. The surfaces of those lesions were smooth, non-scaly, and dusky color. The nodules not tethered to the overlying skin Figure 1.

Provisional diagnosis of kaposi sarcoma was raised but to our surprise the histopathological picture was typical of granulomatous reactions that are seen in sarcoidosis Figure 2.

This finding alerts our attention to look for sarcoidosis. Tuberculin test was negative. Routine hematologic investigations were normal. Serum calcium, liver function tests (LFT), \& renal function tests (RFT) were within the normal level. Nothing was seen by ophthalmological examination. On Chest-XR mild hilar LAP was detected. High resolution CT-Scan showed bilateral diffuse ground glass-masses, and bilateral multiple enlarged hilar and paratracheal LAP. The patient was classified as having stage II sarcoidosis. Accordingly the patient was given $30 \mathrm{mg} /$ day prednisolone for 2 weeks with dramatic clearance of most of the skin lesions Figure 3.

However, 3 months later and suddenly after steroid tapering, the patient develop new, asymptomatic, rapidly growing solitary cutaneous lesion which reached several centimeters in size within only 2 weeks on the right side of his chest, with reactivation of another old lesion on the right leg, this new finding had brought the patient back again to our department. 


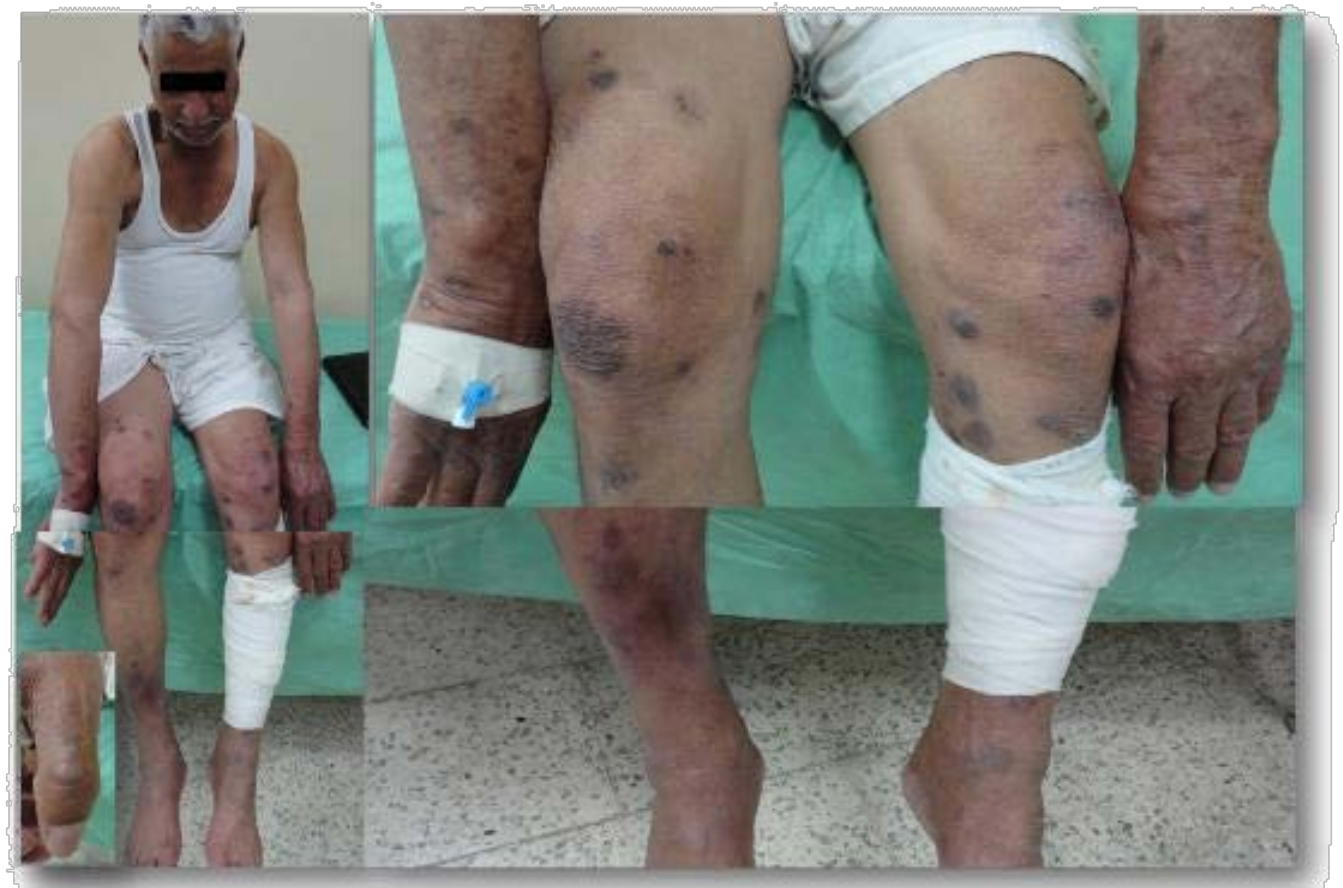

Figure 1. A 60 years old male patient with 3 years history of multiple, diffuse, well-defined brown nodules and plaques over many parts of the body, with smooth, non-scaly, and dusky color.
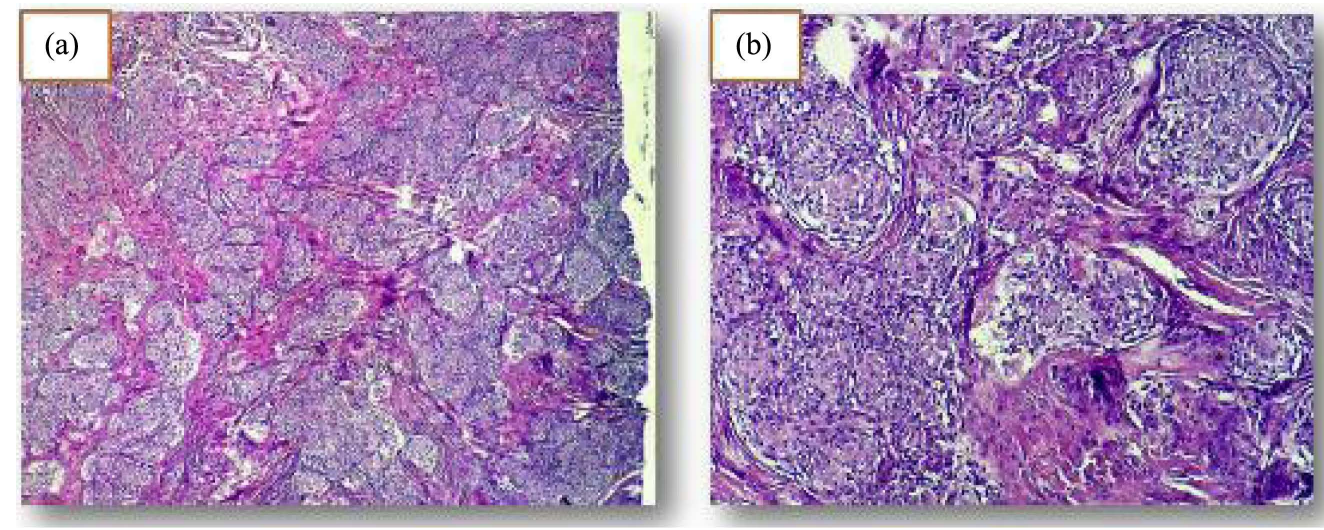

Figure 2. Hematoxylin \& eosin (H \& E) stained section showed typical naked granulomas involving the whole dermis; (a) original magnification $\times 10$; (b) original magnification $\times 40$.

Physical examination revealed solitary, about $5 \mathrm{~cm}$ in diameter, erythematous, smooth surface, arciform, mass located on the lower right axillary line. Another solitary, about $3 \mathrm{~cm}$ in diameter, hyperpigmented, with fine scaly surface, nodule was also found on the lateral aspect of the right leg. No palpable LAP Figure 4 and Figure 5.

Incisional biopsy was taken from the mass on the chest for Histopathological study. Routine hematologic investigations were normal. Liver function tests (LFT), \& renal function tests (RFT) were normal. Chest-XR was normal. Patient asked to repeat the course of steroid therapy (15 mg/day) plus zinc sulphate $440 \mathrm{mg} /$ day until the diagnosis confirmed, and again surprisingly, the mass disappeared completely within 2 weeks of therapy. However, the histopathological study showed diffuse atypical lymphocytic infiltrate of the dermis and subcutaneous fat with frequent mitotic figures a picture of CTCL, but no granuloma was seen Figure 6.

Then shortly after steroid and zinc sulphate weaning, the patient develop sudden eruption of multiple, slightly itchy skin lesions involving the abdomen and thigh. Physical examination showed multiple, erythematous, subcutaneous nodules distributed symmetrically over the abdomen and both thighs, No LAP Figure 7. 


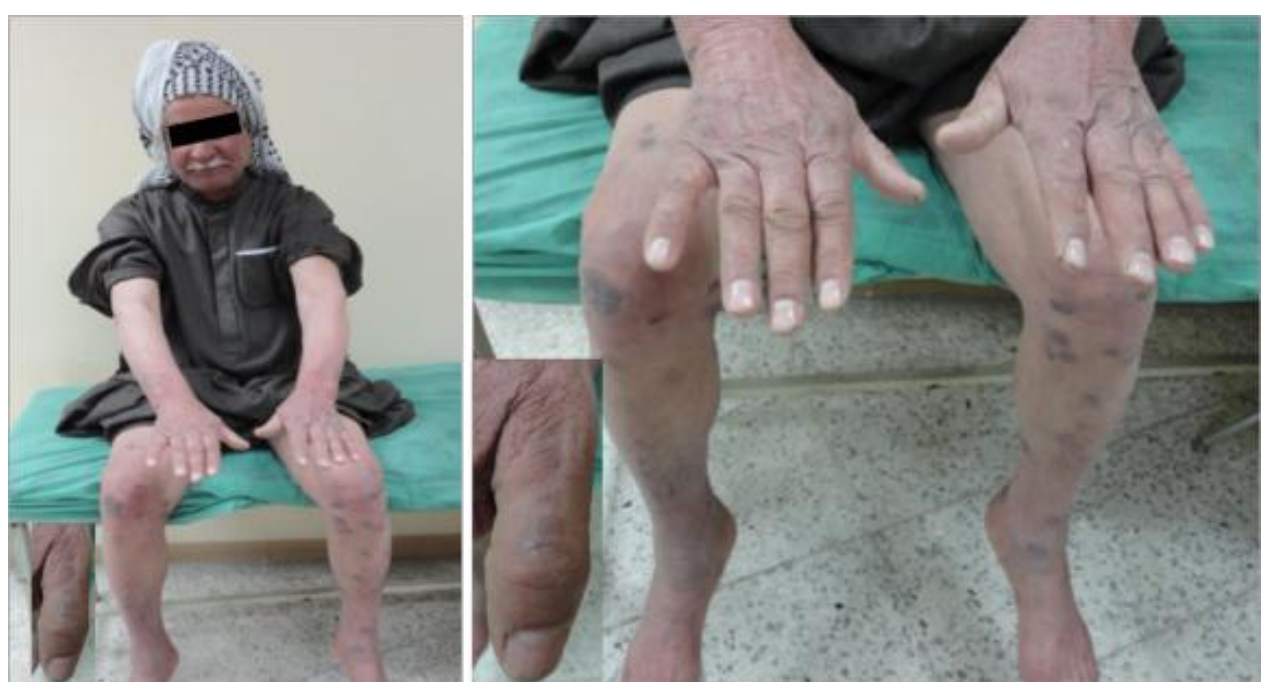

Figure 3. The same patient above after 2 weeks of treatment with $30 \mathrm{mg} /$ day prednisolone with dramatic clearance of most of the skin lesions.

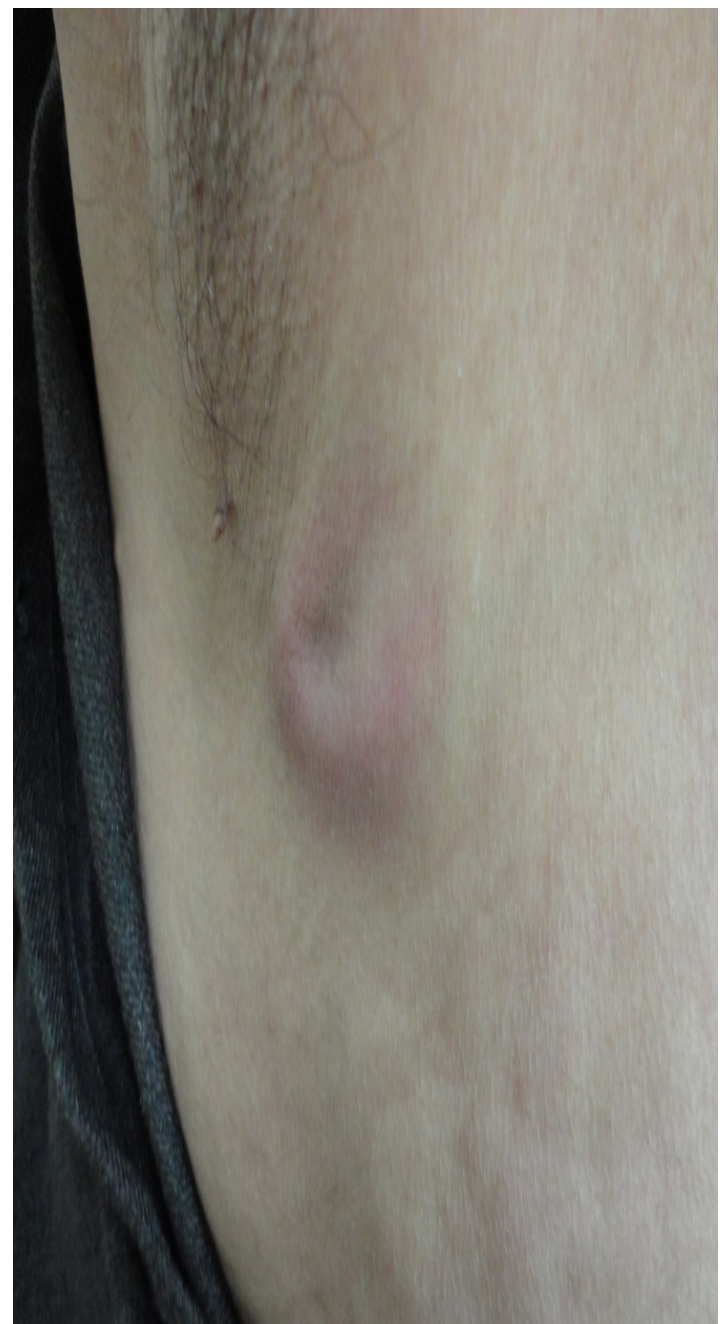

Figure 4. The patient 3 months later developed solitary, about $5 \mathrm{~cm}$ in diameter, erythematous, smooth surface, arciform mass located on the lower right axillary line. 


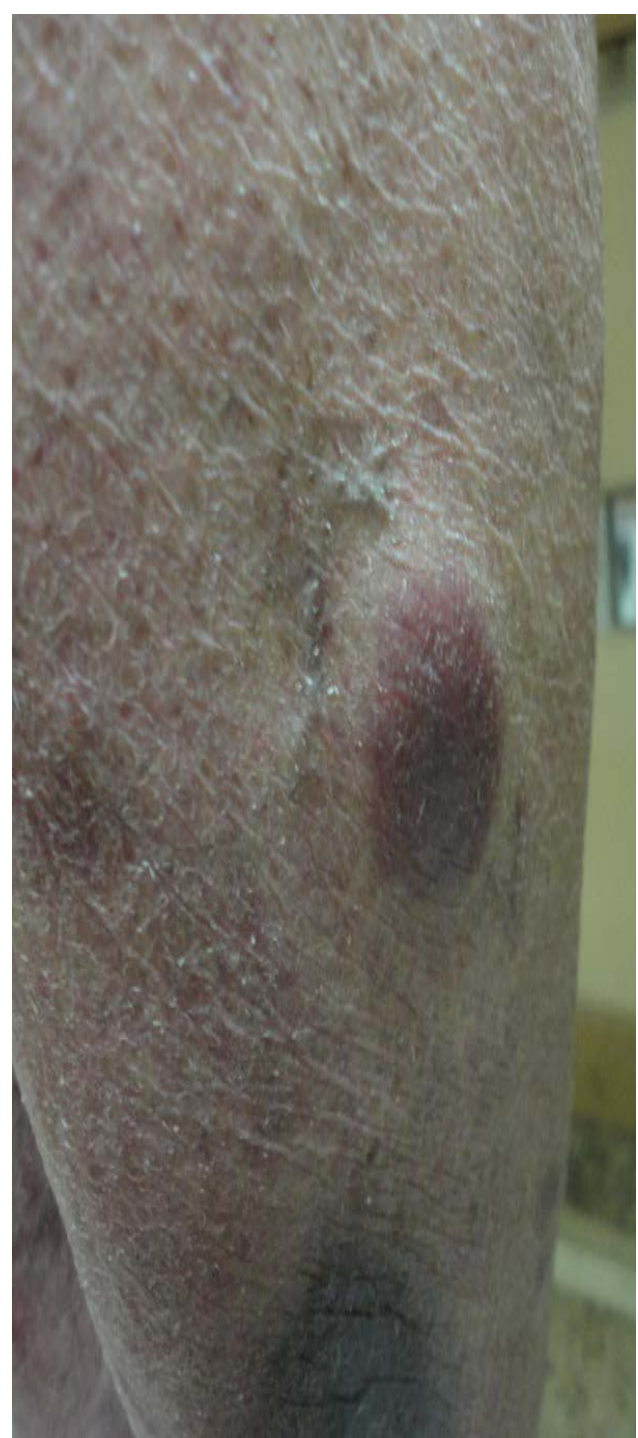

Figure 5. 3 months later developed solitary about $3 \mathrm{~cm}$ in diameter, hyperpigmented, with fine scaly surface nodule on the lateral aspect of right leg.
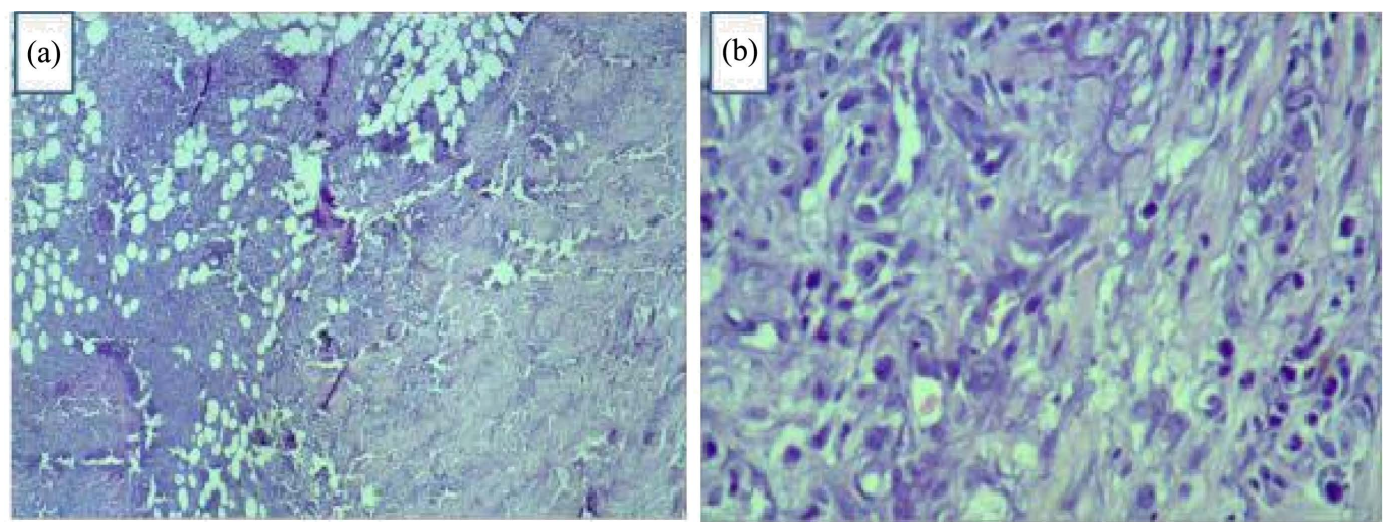

Figure 6. Hematoxylin and eosin (H \& E) stained section showed diffuse atypical lymphocytic infiltration in dermis and subcutaneous fat; (a) original magnification $\times 10$; (b) original magnification $\times 40$. 


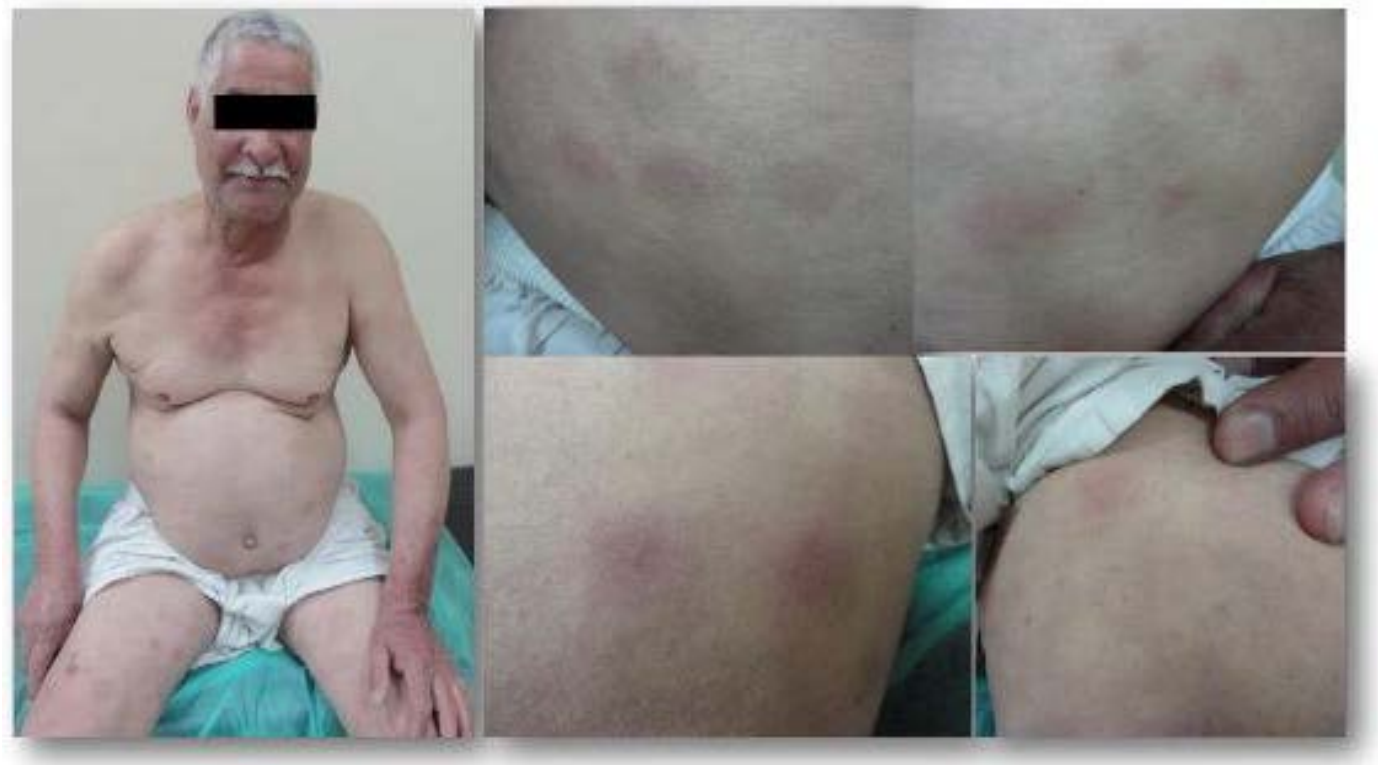

Figure 7. The same patient above after steroid and zinc sulphate weaning, the patient developed multiple, erythematous, subcutaneous nodules distributed symmetrically over the abdomen and both thighs.

Again, biopsy was done, and sent for histopathological study. Routine hematologic, and biochemical investigations were normal. Chest-XR was normal and US showed no abnormality in the abdomen. CT scan of the chest was being arranged. Patient asked to repeat the course of zinc sulphate therapy alone, to prevent masking of the original disease. After 1 month of this regimen patient noticed some improvement but clinically the subcutaneous nodules still there. Biopsy was done again and revealed a picture of diffuse atypical lymphocytic infiltration of dermis and picture goes with T-cell lymphoma.

Consultation to the hematologist was done who decide to make bone marrow aspiration and waiting the result of CT scan to start therapy for CTCL. Bone marrow aspirations reveal no lymphocytic infiltration.

However, while the patient waiting the appointment of CT scan, Patient develop another non tender, rapidly growing skin lesion on his shoulder. By repeating the physical examination we notice large, erythematous, smooth surface mass with irregular nodularity located on the right shoulder, associated with bilateral axillary and left inguinal firm, non tender lymphadenopathy. The patient looks tired, a bit toxic, and feels really ill. Abdominal examination showed for the first time multiple irregular masses but no hepatosplenomegally Figure 8.

Another incisional biopsy was taken from the shoulder mass and showed infiltration of deep dermis and subcutaneous fat by atypical large lymphoid cells with frequent mitosis, and CD markers were positive for CD30, CD43, and ALK-1 which is the marker of large anaplastic T-Cell lymphoma. The result of CT scan of the chest now is ready and showed bilateral axillary and Para-aortic LAP, with increased thickness of the abdominal wall Figure 9.

The final diagnosis now was established as the patient having large anaplastic T-Cell lymphoma, and accordingly chemotherapy was started, but unfortunately, patient condition rapidly deteriorated and patient died after 2 weeks of the initial chemotherapy cycle.

\section{Discussion}

Sarcoidosis in Iraq is a very rare and even the reported cases are not well documented. Accordingly we are very surprised to diagnose this patient as a case of sarcoidosis, while lymphoma is a common disease encountered in clinical practice. So from reviewing the literatures and the finding in the reported previous cases we can think that no proper cases of sarcoidosis seen and all patients react to same antigens probably virus that overtime change either into sarcoid reaction first and then develop to lymphoma, or lymphoma appear first and sarcoid reaction appear during the course of the disease [13]. Accordingly, we can hypothesize that there are no pure cases of sarcoidosis and all the reported patients with sarcoid, are either remain as a sarcoidosis for years or transfer rapidly to a stage of lymphoma. 


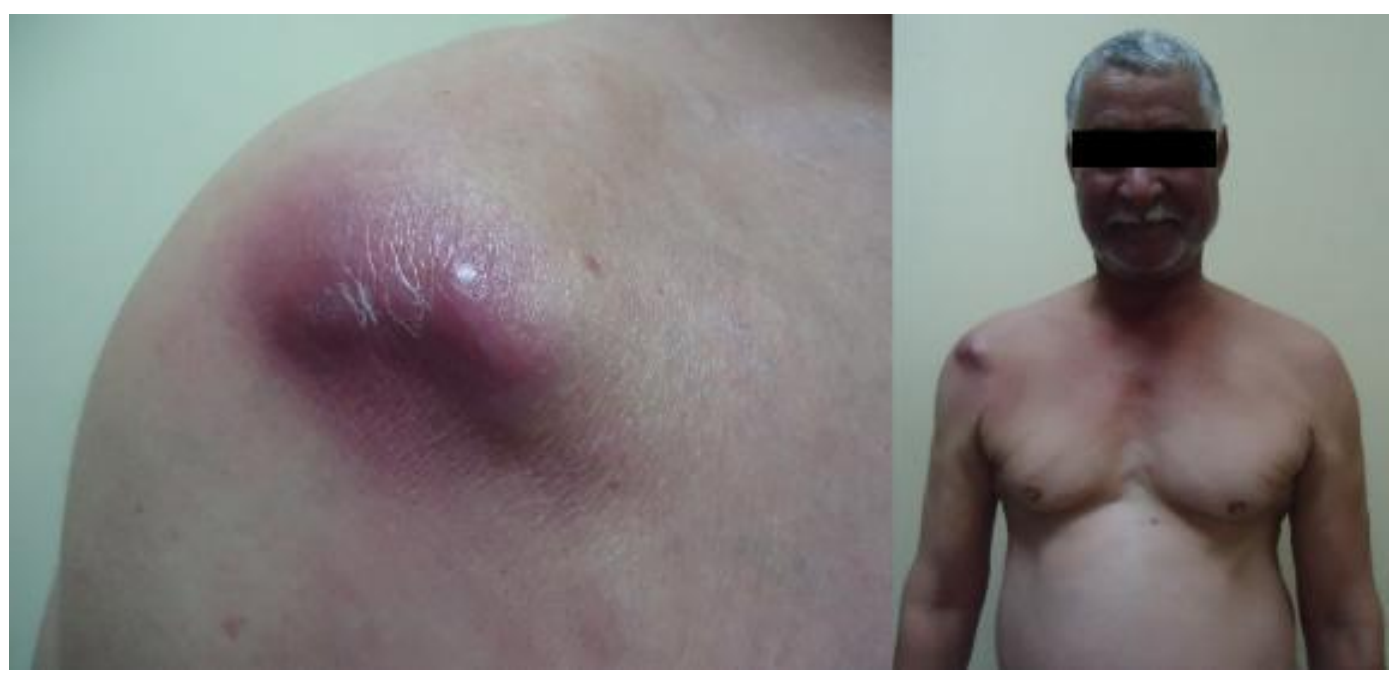

Figure 8. The same patient above developed Large, erythematous, smooth surface mass with irregular nodularity located on the right shoulder.
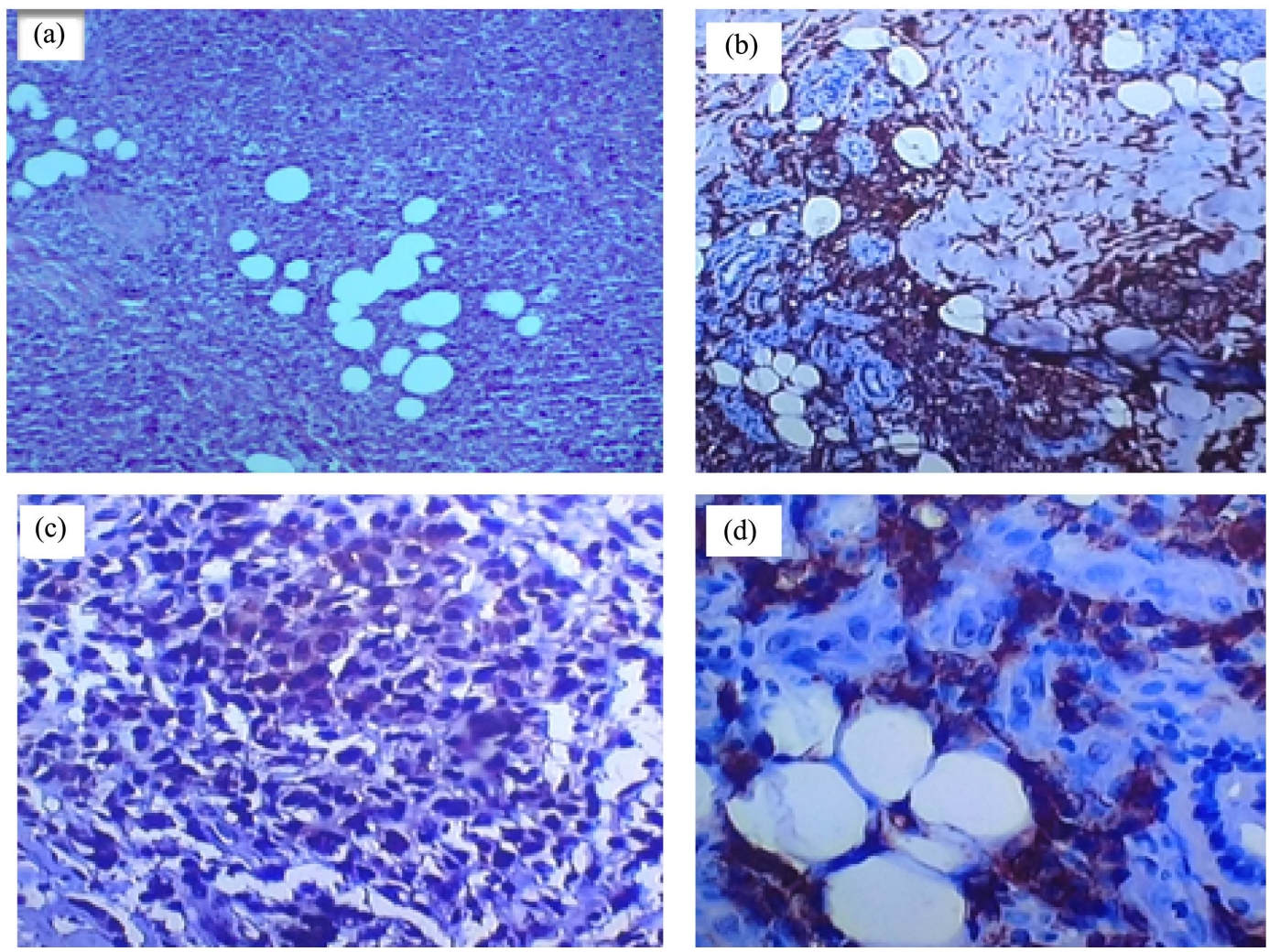

Figure 9. Hematoxylin and eosin (H \& E), CD markers stained section showed infiltration of deep dermis and subcutaneous fat by atypical large lymphoid cells with frequent mitosis. (a) H \& E original magnification $\times 10$; (b) CD43 original magnification $\times 10$; (c) CD30 original magnification $\times 10$; (d) ALK-1 original magnification $\times 40$.

So we can suggest that there is a state of spectrum with two poles one is the sarcoidosis and the other is lymphoma and there are mixed cases with overlapping features in the middle and all sharing same etiopathological factors in which viruses are the most incriminated agents.

In countries where sarcoidosis is a common problem every doctor should be aware about the spectrum of sar- 
coidosis-lymphoma state otherwise either the pole of the spectrum will be missed and wrongly managed. Further researches in this field are mandatory in order to reach out the final decision of the strange clinical and pathological conditions.

\section{Conclusion}

In conclusion, we agree to use the term ("sarcoidosis-lymphoma syndrome") or ("lymphoma-sarcoidosis syndrome") to all cases of so called sarcoidosis/lymphoma and watch the patient by frequent examination, histopathological testing, and doing CD markers in order to detect any further change from one pole to another one.

\section{Disclosure}

This study is an independent study and not funded by any of the drug companies.

\section{References}

[1] Joint Statement of the American Thoracic Society (ATS), the European Respiratory Society (ERS) and the World Association of Sarcoidosis and Other Granulomatous Disorders (WASOG) (1999) Statement on Sarcoidosis. American Journal of Respiratory and Critical Care Medicine, 160, 736-755.

[2] McGrath, D.S., Goh, N., Foley, P.J. and duBois, R.M. (2001) Sarcoidosis: Genes and Microbes-Soil or Seed. Sarcoidosis Vasculitis Diffuse Lung Disease, 18, 149-164.

[3] King, C.S. and Kelly, W. (2009) Treatment of Sarcoidosis. Disease-A-Month, 55, 704-718. http://dx.doi.org/10.1016/j.disamonth.2009.06.002

[4] Nunes, H., Bouvry, D., Solar, P. and Valeyre, D. (2007) Sarcoidosis. Orphanet Journal Rare Disease, 2, 46. http://dx.doi.org/10.1186/1750-1172-2-46

[5] Sadek, M.M., Yung, D., Birnie, D.H., Beanlands, R.S. and Nery, P.B. (2013) Corticosteroid Therapy for Cardiac Sarcoidosis: A Systemic Review. Canadian Journal of Cardiology, 29, 1034-1041. http://dx.doi.org/10.1016/j.cjca.2013.02.004

[6] Goswami, T., Siddique, S., Cohen, P. and Cheson, B.D. (2010) The Sarcoid-Lymphoma Syndrome. Clinical Lymphoma, Myeloma \& Leukemia, 4, 241-247.

[7] Brincker, H. (1986) The Sarcoidosis-Lymphoma Syndrome. British Journal Cancer, 54, 467-473. http://dx.doi.org/10.1038/bjc.1986.199

[8] Brinker, H. and Wilbek, E. (1974) The Incidence of Malignant Tumours in Patients with Respiratory Sarcoidosis. British Journal Cancer, 29, 247-251. http://dx.doi.org/10.1038/bjc.1974.64

[9] Brincker, H. (1995) Sarcoidosis and Malignancy. Chest, 108, 1472-1474. http://dx.doi.org/10.1378/chest.108.5.1472

[10] William, D., Timothy, G. and Dirk, M. (2011) Sarcoidosis in Andrews Disease of the Skin, Clinical Dermatology. 11th Edition, Saunders Alsevier, 31, 700-707.

[11] Karakantza, M., Matutes, E., MacLennan, K., et al. (1996) Association between Sarcoidosis and Lymphoma Revisited. Journal Clinical Pathology, 49, 208-212. http://dx.doi.org/10.1136/jcp.49.3.208

[12] Cohen, P.R. and Kurzrock, R. (2007) Sarcoidosis and Malignancy. Clinical Dermatology, 25, 326-323. http://dx.doi.org/10.1016/j.clindermatol.2007.03.010

[13] Maayan, H., Ashkenazi, Y., Nagler, A. and Izbicki, G. (2011) Sarcoidosis and Lymphoma: Case Series and Literature Review. Sarcoidosis Vascular Diffuse Lung Disease, 28, 146-152. 